\title{
Gait Analysis: Normal and Pathological Function
}

Editors: Jacquelin Perry and Judith M. Burnfield

Bibliographic Data: ISBN: 978- 1556427664, 2010, Slack Incorporated, New Jersey, \$92.95, 551pages, hardcover

Subjects: Gait physiology, biomechanics of gait, gait analysis.

DESCRIPTION: This text covers the work of Dr Jacquelin Perry in her years as a medical professional focusing on the human gait with extensive contribution from Judith M. Burnfield, PhD, PT.

PURPOSE: The aim of this second edition is to analyze gait further by bringing the relevant data up to date and covering current research, more sophisticated methods, and the latest equipment available.

FEATURES: The text is broken down into six sections as follows: I- Fundamentals which is divided into 3 chapters: 1, Gait Cycle; 2, Phases of gait; 3, Basic Functions. Section II, Normal Gait, covers chapters 4 to 9: 4, AnkleFoot Complex; 5, Knee; 6, Hip; 7, Head, Trunk, and Pelvis; 8, Arm; 9, Total Limb Function and Bilateral Synergistic Relationships.

Section III, Pathological Gait, is from chapter 10 to 14: 10, Pathological Mechanisms; 11, Ankle and Foot Gait Deviations; 12, Knee Gait Deviations; 13, Hip Gait Deviations; 14, Trunk and Pelvis Gait Deviations.
Clinical Considerations are discussed in Section IV: Chapter 15, Examples of Pathologic Gait; 16, Pediatric Gait Analysis. Section V is for Advanced Locomotor Function in chapter 17, Stair Negotiation an in 18, Running.

In section VI Quantified Gait Analysis is presented: Chapter 19, Gait Analysis Systems; 20, Motion Analysis; 21, Muscle Control and Dynamic Electromyography; 22, Kinetics of Gait: Ground Reaction; Forces, Vectors, Moments, Power, and Pressure; 23, Stride Analysis; 24, Energy Expenditure

AUDIENCE: Orthopaedic, physical therapy and other rehabilitation professionals who are involved in musculoskeletal patient care could easily use this is book as a reference text. It can also be useful in designing some athletic training programmes, physical therapy programmes and gait workshops.

ASSESSMENT: This is an extremely informative book and an absolute asset for anyone who is studying and/or dealing with physio-pathological lower limb biomechanics.

Reviewed by: Fadil Özyener MD, PhD, Uludag University Medical School, Bursa Turkey 